\title{
A FLAVORED NEUTRINO BEAMS FACILITY
}

S. Mori and J. K. Walker

March 31, 1980

\section{Introduction}

We have considered a $1 \mathrm{TeV}$ facility in the neutrino area which would produce a high flux of $\nu_{e}$ and $\nu_{T}$ type neutrinos. In the case of the $\nu_{e}$, we investigated two possibilities. These are (1) a special $K_{L}^{0}$ beam located upstream of Lab $E$ and using the decay.

$$
\mathrm{K}_{\mathrm{L}}^{0} \rightarrow \pi^{-} \mathrm{e}^{+} \mathrm{\nu}_{\mathrm{e}} \text { and } \pi^{+} \mathrm{e}-\bar{\nu}_{\mathrm{e}} \quad(B R=39 \%)
$$

as the source of the neutrinos and (ii) a dump located upstream of lab $E$ and using the decay

$$
\mathrm{D}(1.86) \rightarrow \mathrm{Ke}^{+} \nu_{\mathrm{e}} \text { and } \mathrm{Ke}^{-} \bar{\nu}_{\mathrm{e}}
$$

as the source of neutrinos. We conclude that in both cases, the available flux of electron type neutrinos is increased by about one order of magnitude in the region of the $15^{\prime}$ B.C. and Lab C compared to the previous design of a $\nu_{e}$ beam using the existing beam pipe with a $K_{L}^{0}$ beam (TM-769). In the case of the $K_{L}^{0}$ facility, however, there is a substantial background of muon type neutrinos coming principally from the products of neutral hyperon decay. The $\mathrm{K}_{\mathrm{L}}^{0}$ beam is expensive to construct and operate and requires a substantial number of large magnets to minimize background from charged particle and neutral hyperon decay. For these reasons, we regard the beam dump as the best facility for a $\nu_{e}$ beam. For $\nu_{\tau}$ type neutrinos, we have considered the production and decay of $F$ type mesons 


$$
\begin{aligned}
\mathrm{F}^{+} \rightarrow \nu_{\tau}+\tau^{+} & \\
\tau^{ \pm} & \rightarrow \bar{\nu}_{\tau}+l^{+}+\bar{\nu}_{\mathrm{e}}\left(l^{+}=\mathrm{e}^{+} \text {or } \mu^{+}\right) \\
& \rightarrow \bar{\nu}_{\tau}+\pi^{+} \\
& \rightarrow \bar{\nu}_{\tau}+\rho^{+}
\end{aligned}
$$

B.R.

$3 \%$

$36 \%$

$10 \%$

$20 \%$

in a beam dump located upstream of Lab E. The fluxes appear to be adequate to not only detect the existence of the $\nu_{\tau}$ directly, but carry out a study of its properties. For these reasons, we conclude that the upgrade of the neutrino facilities for operation at $1 \mathrm{TeV}$ should include a beam dump facility for the provision of intense $\nu_{e}$ and $\nu_{\tau}$ beams in the detectors in that area.

II. $K_{L}^{0}$ Beam

In Figure 1 we show the physical layout of the neutrino area in the region under consideration. The length of $\mathrm{K}_{\mathrm{L}}^{0}$ decay region should be long to maximize neutrino flux and yet not so long that it substantially compromises the shielding action of the berm for normal running of $\nu_{\mu}$ type neutrinos. We have considered decay lengths in the range $130-250 \mathrm{~m}$. The distance from the end of the decay region to the detector should be minimized for flux considerations and we have chosen $190 \mathrm{~m}$ as the distance to Lab C. This distance will depend ultimately on the design of the active muon shield which is required upstream of Lab $E$. The present design permits $60 \mathrm{~m}$ for the combined dump and shield function. We do not expect our conclusions to depend sensitively on expected changes of that number. Fluxes have been calculated at the front of Lab $C$ for a detector of radius $1.5 \mathrm{~m}$ and using the Monte Carlo program described in TM-769.

The $\nu_{e}\left(\bar{v}_{e}\right)$ flux is determined by the decay pipe radius and the angular acceptance of the magnets and collimators used to make the beam. 
Figure 2 shows the $\nu_{\mathrm{e}}$ flux versus the decay pipe radius for various neutrino energies. On this basis, we have chosen the easily available pipe of $0.457 \mathrm{~m}$ radius. Figure 3 shows the $\nu_{e}$ flux versus the angular acceptance of the beam for various neutrino energies. An angular acceptance of $\geq \pm 5 \mathrm{mr}$ is clearly desirable and we have proceeded with this criterion. The $\nu_{\mathrm{e}}$ flux for $400 \mathrm{GeV}$ and $1000 \mathrm{GeV}$ incident protons is shown in Figure 4. For comparison the $\nu_{e}$ flux from $K_{L}^{0}$ in the existing decay pipe is shown and is seen to be an order of magnitude inferior in intensity.

Backgrounds of muon neutrinos from decay of long lived charged particles ( $\tau>10^{-10}$ secs) can be reduced by sweeping magnets immediately downstream of the target. These backgrounds have been discussed at length by S.Mori in TM-769. In Figure 5, we show a magnet configuration which provides some suppression of this background using mostly existing magnets (some with increased apertures) to match the required acceptance of the neutral beam. The total background flux is shown in Figure 6 at $400 \mathrm{GeV}$ and has been described in TM-769. It can be seen that the background of $\nu_{\mu}$ and $\bar{v}_{\mu}$ is severe and is worse in the case of the new $K_{L}^{0}$ beam than that in the existing decay pipe (see TM-769). At $1000 \mathrm{GeV}$, the situation is worse still and is shown in Figure 7 . The $\bar{v}_{\mu}$ background at $1000 \mathrm{GeV}$ would lead to very large substractions and consequently large errors in experiments which did not identify the type of incident neutrino. The basic reason for the higher backgrounds is that these neutrinos come from short-lived sources (principally hyperons) which almost all decay independent of the length of the decay region, whereas the $K_{L}^{0}$ now have only $130 \mathrm{~m}$ compared with the existing $400 \mathrm{~m}$ decay pipe. 
We considered increasing the length of the decay region for the $K_{L}^{0}$ beam. Changing the decay length from $130 \mathrm{~m}$ to $250 \mathrm{~m}$ produces a $10 \%$ increase in $\nu_{\mathrm{e}}$ flux at $25 \mathrm{GeV}$ and $50 \%$ at $100 \mathrm{GeV}$. The signal to background is improved by the same amount but is still much less than desirable. In addition, the function of the berm as a shield for normal wide-band beam running at 1 TeV has been compromised.

An alternative way to reduce background is to install many more sweeping magnets over the entire length $(130 \mathrm{~m})$ of the decay pipe. However, due to the required aperture of $\pm 5 \mathrm{mr}$ to achieve the high intensity of the neutral beam, the gap size of these sweeping magnets becomes prohibitive (1.0m at $100 \mathrm{~m}$ distance from the target).

We are, therefore, forced to the conclusion that the new $K_{L}^{0}$ beam is a high flux source of $\nu_{e}$, however, there is no simple economical solution to achieve an acceptably low background of $\nu_{\mu}$ type neutrinos.

III. Beam Dump Source of $v_{e}$ Beam

The study of the prompt production of leptons in hadron interactions has progressed substantially in the last year. A study of prompt neutrino production by three experimental groups at CERN, and a study of prompt muon production at Fermilab have been interpreted in terms of $D$ meson production and subsequent semi-leptonic decay. The cross sections for $D$ production from these four experiments are shown in figure 8 . These cross sections were deduced on the assumption that the cross sections depend linearly on the atomic number $A$, the semi-leptonic branching ratio is $10 \%$ and assuming different models of the $x$ and $p_{\perp}$ dependence of the production process. The different assumptions of the models may introduce uncertainties in the final cross sections by as much as a factor of 2 . 
From Figure 8 we take a $D(\bar{D})$ cross production section of $17 \mu$ barns $400 \mathrm{GeV}$ for the purpose of making prompt neutrino flux estimates. In addition, we assume that the cross section for $D(\bar{D})$ production will increase to $34 \mu$ barns at an incident proton energy of $1000 \mathrm{GeV}$. Electron neutrino energy distributions from the $D$ decay and the $x$ and $P_{\perp}$ distributions for D production were taken from Hinchliffe and C.M.L.Smith. The $D \rightarrow K \nu_{e} e^{+}$branching ratio was taken to be $10 \%$. The cross section for charm production was assumed to depend linearly on $A$, the atomic number. The design of the primary proton beam transport will permit the beam size to be as large as 1 "diameter when it strikes the dump thereby al lowing the use of tungsten as the dump material. The $\nu_{e}$ and $\bar{v}_{e}$ fluxes were calculated using the Monte Carlo program used in TM-802 and are shown in Figure 9. There are equal numbers of electron and muon neutrinos from D decay. No estimate of the additional contribution of muon neutrinos from $\pi$ and $\mathrm{K}$ decay has been made. However, based on the beam dump experiments at CERN and FNAL, it appears that their background wil1 be less in the case of a dump source than that estimated in the previous section for a $K_{L}^{0}$ beam. In addition, it is likely that these backgrounds will decrease faster than the prompt neutrinos at finite production angles.

The flux of electron type neutrinos is substantial and compares well with that obtained from the $K_{L}^{0}$ source described in section II. Given that the expected muon type neutrino background is expected to be less in this arrangement than from the $\mathrm{K}_{\mathrm{L}}^{0}$ beam, we consider the dump source as the more attractive from a physics point of view. It is clear that this choice is also more economical than the $K_{L}^{0}$ beam. 
In Figures 10 and 11 , we show the neutrino flux expected in the various detector buildings for a dump constructed $60 \mathrm{~m}$ upstream of Lab E for 400 and $1000 \mathrm{GeV}$ operation. There is a factor of 10 difference in the low energy neutring flux and relatively little difference about $100 \mathrm{GeV}$. Table I shows relative interaction rates of $v_{e}$ type neutrinos in a given mass detector located in the different buildings. Because of the cross section rising with energy, there is a relatively mild dependence of event rate as a function of detector distance from the dump.

TABLE I

$\nu_{e}\left(\bar{\nu}_{e}\right)$ Relative Rates In Same Detector As A Function of Distance From Dump

\begin{tabular}{r|c|c|c|c} 
& Lab E (60m) & $15^{\prime}$ BC (125m) & Lab C (190m) & Lab X (250m) \\
\hline $400 \mathrm{GeV}$ & 0.43 & 0.27 & 0.17 & 0.1 \\
$1000 \mathrm{GeV}$ & 1.54 & 1.24 & 1.0 & 0.71 \\
\hline
\end{tabular}

The predicted angular distribution of prompt $\nu_{e}\left(\bar{\nu}_{e}\right)$ is shown in Figures 12 and 13 for 400 and $1000 \mathrm{GeV}$ respectively. IV. Beam Bump Source of $\nu_{\tau}$ Beam

S.Mori in TM-848 made preliminary estimates of $\nu_{\tau}$ fluxes which could be obtained from a beam dump. The Monte Carlo program and many detailed assumptions were outlined in that Technical Memo and here we comment only on the different assumptions which we have made at this time.

1. We assume the integrated cross section of the $F$ pair cross section per nucleon is $25 \%$ of the corresponding $D$ cross section, namely is $4.25 \mu \mathrm{b}$ at $400 \mathrm{GeV}$ and rises to twice that at $1000 \mathrm{GeV}$. In TM-848 a figure of $10 \mu \mathrm{b}$ independent of energy was assumed.

2. The cross section of $F$ pair production is assumed to vary linearly with $A$ rather than as $A^{2 / 3}$ which was assumed in TM- 848 . 
3. The beam dump is assumed to be made of tungsten. The new calculated flux of $\nu_{\tau}$ neutrinos is shown in Figure 14 for $400 \mathrm{GeV}$ incident protons in each of the various laboratories. The corresponding fluxes for $1000 \mathrm{GeV}$ protons is shown in Figure 15. Table II shows the relative interactions rates of $\nu_{\tau}$ type neutrinos in a given mass detector located in the different buildings. Because we assume that the cross section rises with energy, there is a relatively mild dependence of event rate as a function of detector distance from the dump.

$$
\begin{aligned}
& \nu_{\tau}\left(\bar{\nu}_{\tau}\right) \text { Relative Rates in Same Detector } \\
& \text { As a Function of Distance From Dump }
\end{aligned}
$$

\begin{tabular}{r|c|c|c|c} 
& $\operatorname{Lab} E(60 \mathrm{~m})$ & $15^{\prime} \mathrm{BC}(125 \mathrm{~m})$ & $\operatorname{Lab} C(190 \mathrm{~m})$ & $\operatorname{Lab} X(250 \mathrm{~m})$ \\
\hline $400 \mathrm{GeV}$ & 0.46 & 0.3 & 0.19 & 0.1 \\
$1000 \mathrm{GeV}$ & 1.68 & 1.34 & 1.0 & 0.68 \\
\hline
\end{tabular}

Prompt electron and muon neutrinos produced in the dump from charm production described in Section III willconstitute a major background to experiments searching for $\nu_{\tau}$ interactions. The ratio of interaction rates for $\nu_{\tau}$ and $v_{e}$ type neutrinos in Lab C at $1000 \mathrm{GeV}$ is calculated to be 0.1 . This gives a measure of the background rejection that is required for any detector of $\nu_{\tau}$ type neutrinos.

Figure 16 and 17 show the expected angle dependence of the $\nu_{\tau}$ flux at $400 \mathrm{GeV}$ and $1000 \mathrm{GeV}$. The angle range covers essentially the entire forward hemisphere i.e. out to $90 \mathrm{cms}$ at $1000 \mathrm{GeV}$ incident proton energy. The facility should be constructed to explore the full range of production angles. This will permit a study of prompt neutrino production where $\pi$ and $K$ decay neutrinos are suppressed (TM-848). In addition, we expect the large angle region to be the source of neutrinos or other neutrino-like 
particles coming from the decay of high mass particles.

\section{Proton Beam Transport}

In the construction of the beam dump neutrino beam it is vitally important to eliminate other sources of neutrinos. A major potential problem in this regard is scraping of the halo of the proton beam in its transport towards the dump. To minimize this effect, it is possible to collimate the proton beam in the pre-hall area when the beam is pointing we11 above the detectors. Ionization counters can be used along the beam transport as a monitor of proton losses.

\section{Summary}

We have shown that a beam dump facility operated at 1 TeV will provide an intense beam of $\nu_{e}$ and $\nu_{\tau}$ type neutrinos in the neutrino area at Fermilab. Fluxes in locations of the various detectors have been presented as a function of energy and angle. The dump should be constructed to accept protons from 400 to $1000 \mathrm{GeV}$ in the angle range 0 to 40 milleradians. 
Figure 1 Schematic of the layout of the $K_{L}^{0}$ beam relative to the detector buildings in the neutrino laboratory.

Figure 2 Electron neutrino flux versus decay pipe radius for various energies.

Figure 3 Electron neutrino flux versus angular acceptance for various energies.

Figure 4 Electron neutrino flux at 400 and $1000 \mathrm{GeV}$ averaged over a $1.5 \mathrm{~m}$ radius detector in Lab $C$. Also shown is the $\nu_{e}$ flux from a $K_{L}^{0}$ beam in the existing decay pipe.

Figure 5 Magnet configuration for the $\mathrm{K}_{\mathrm{L}}^{0}$ beam.

Figure 6 Electron neutrino flux at $400 \mathrm{GeV}$ in Lab C. Also shown is the $\nu_{\mu}\left(\bar{\nu}_{\mu}\right)$ background from the decay of charged and neutral particles as described in the text.

Figure 7 Electron neutrino flux at $1000 \mathrm{GeV}$ in Lab C. Also shown is the $\nu_{\mu}\left(\bar{\nu}_{\mu}\right)$ background from the decay of charged and neutral particles as described in the text.

Figure 8 Measured cross sections for charm particle production at Fermilab and CERN. The various experiments are identified on the figure and their associated measurements.

Figure 9 Electron neutrino flux in Lab $C$ averaged over a $1.5 \mathrm{~m}$ rad detector at 400 and $1000 \mathrm{GeV}$.

Figure 10 Electron neutrino flux in each detector building at $400 \mathrm{GeV}$.

Figure 11 Electron neutrino flux in each detector building at $1000 \mathrm{GeV}$.

Figure 12 Predicted angular distribution of prompt electron neutrinos at $400 \mathrm{GeV}$.

Figure 13 Predicted angular distribution of prompt electron neutrinos at $1000 \mathrm{GeV}$.

Figure 14 Flux of $\nu_{\tau}\left(\bar{\nu}_{\tau}\right)$ averaged over a $1.5 \mathrm{~m}$ radius detector in each detector building at $400 \mathrm{GeV}$.

Figure 15 Flux of $\nu_{\tau}\left(\bar{\nu}_{\tau}\right)$ averaged over a $1.5 \mathrm{~m}$ radius detector in each detector building at $1000 \mathrm{GeV}$. 
Figure 16 Predicted angular distribution of tau neutrinos at $400 \mathrm{GeV}$. Figure 17 Predicted angular distribution of tau neutrinos at $1000 \mathrm{GeV}$. 
TM-953

2ं.51.000

Page 11

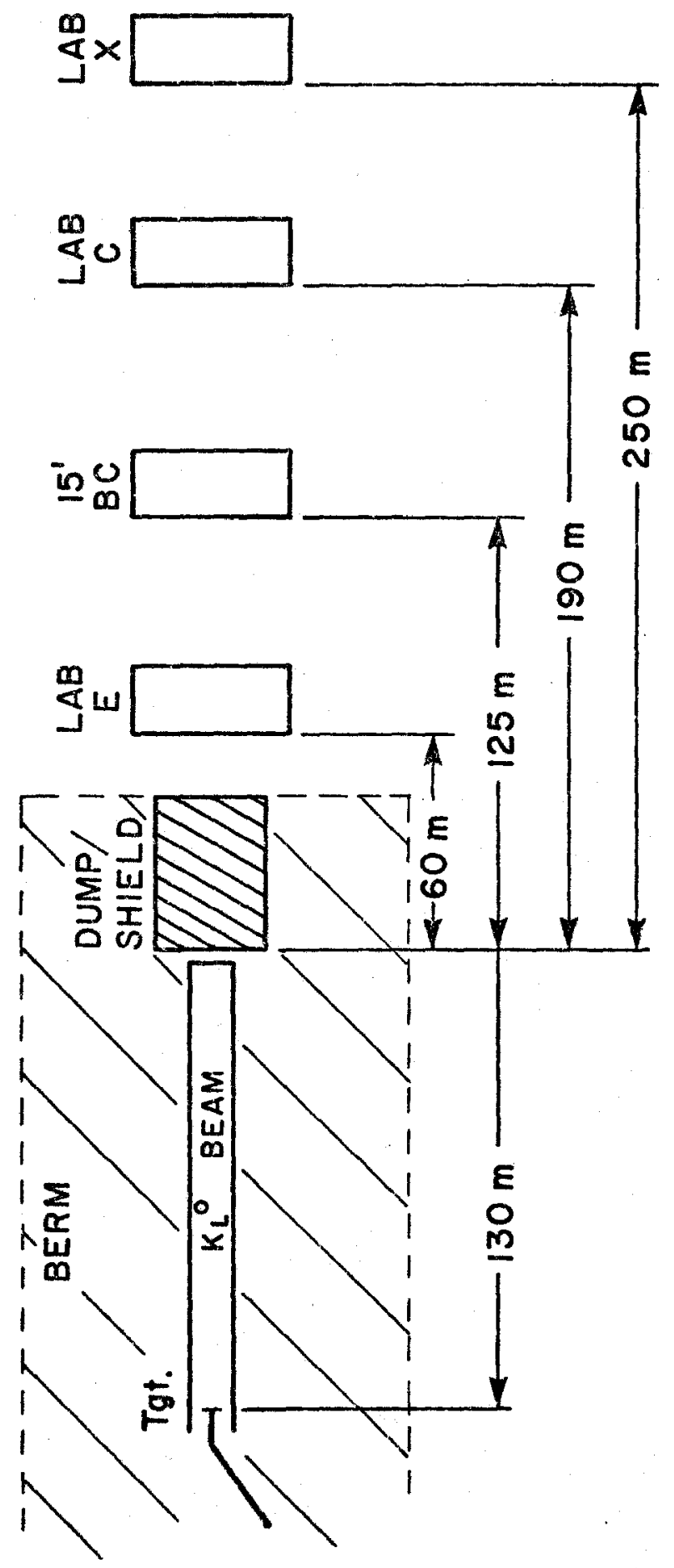

0
$\frac{1}{3}$
$\frac{10}{4}$ 
Figure 2

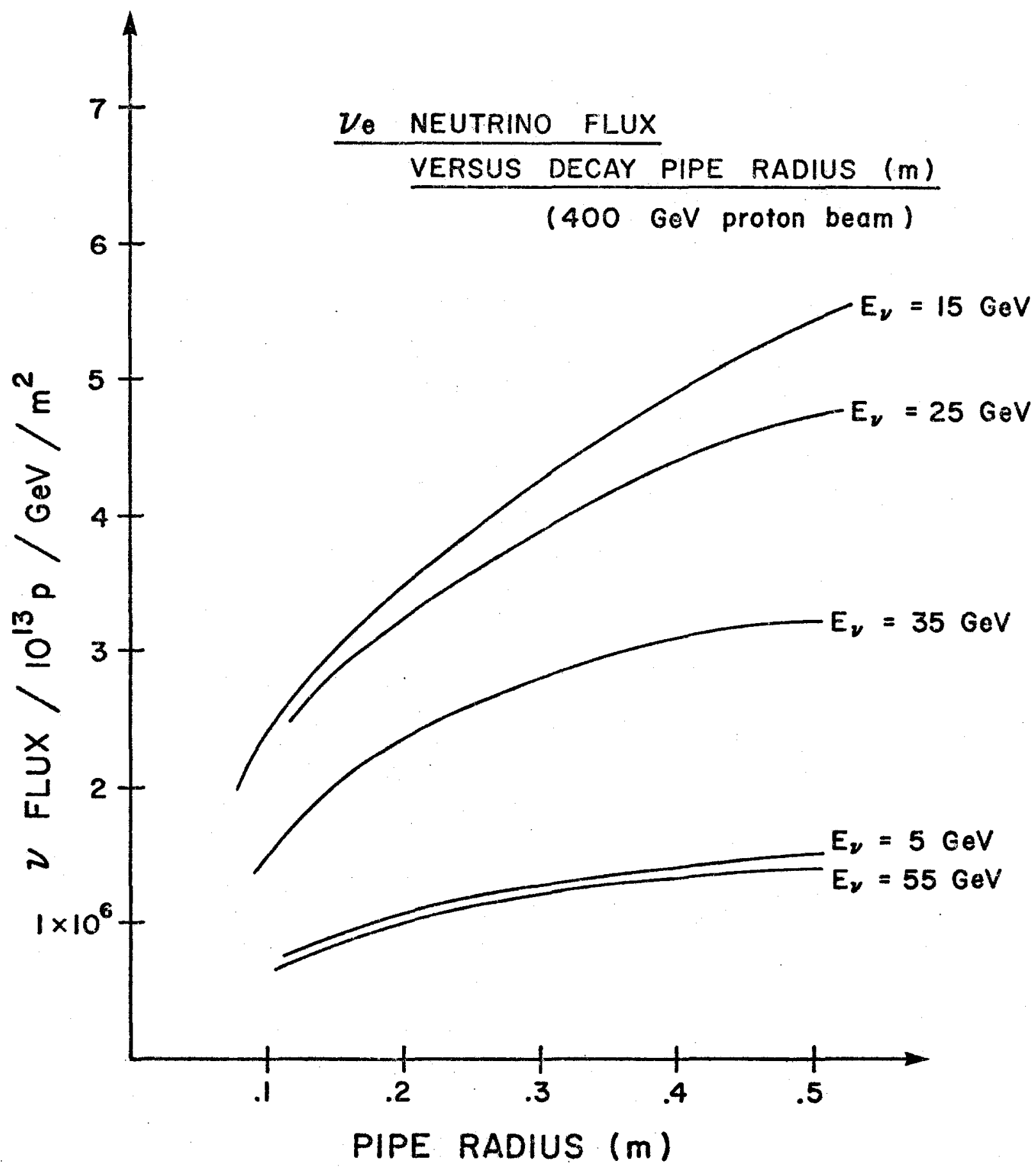




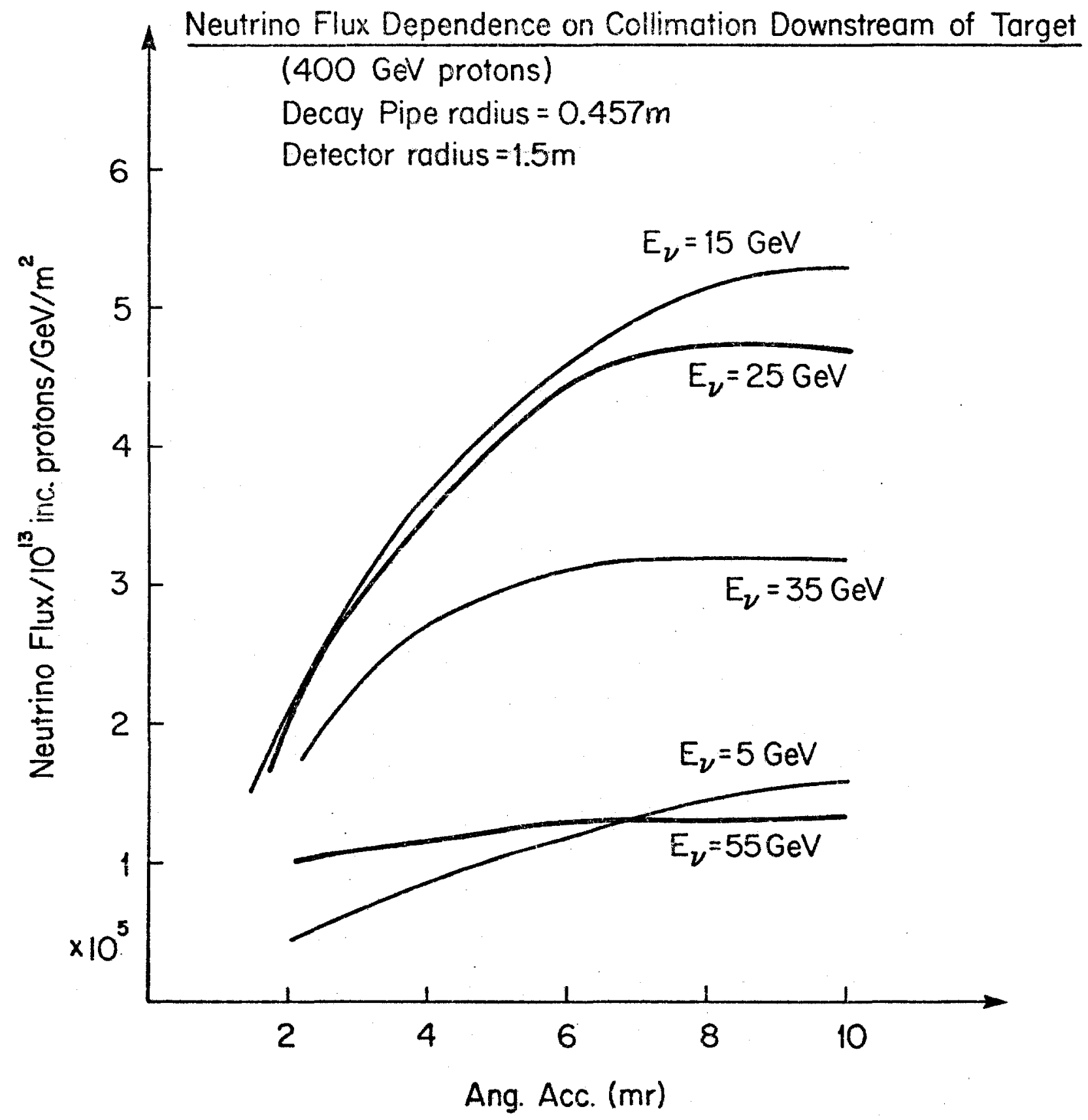

Figure 3 
Figure 4

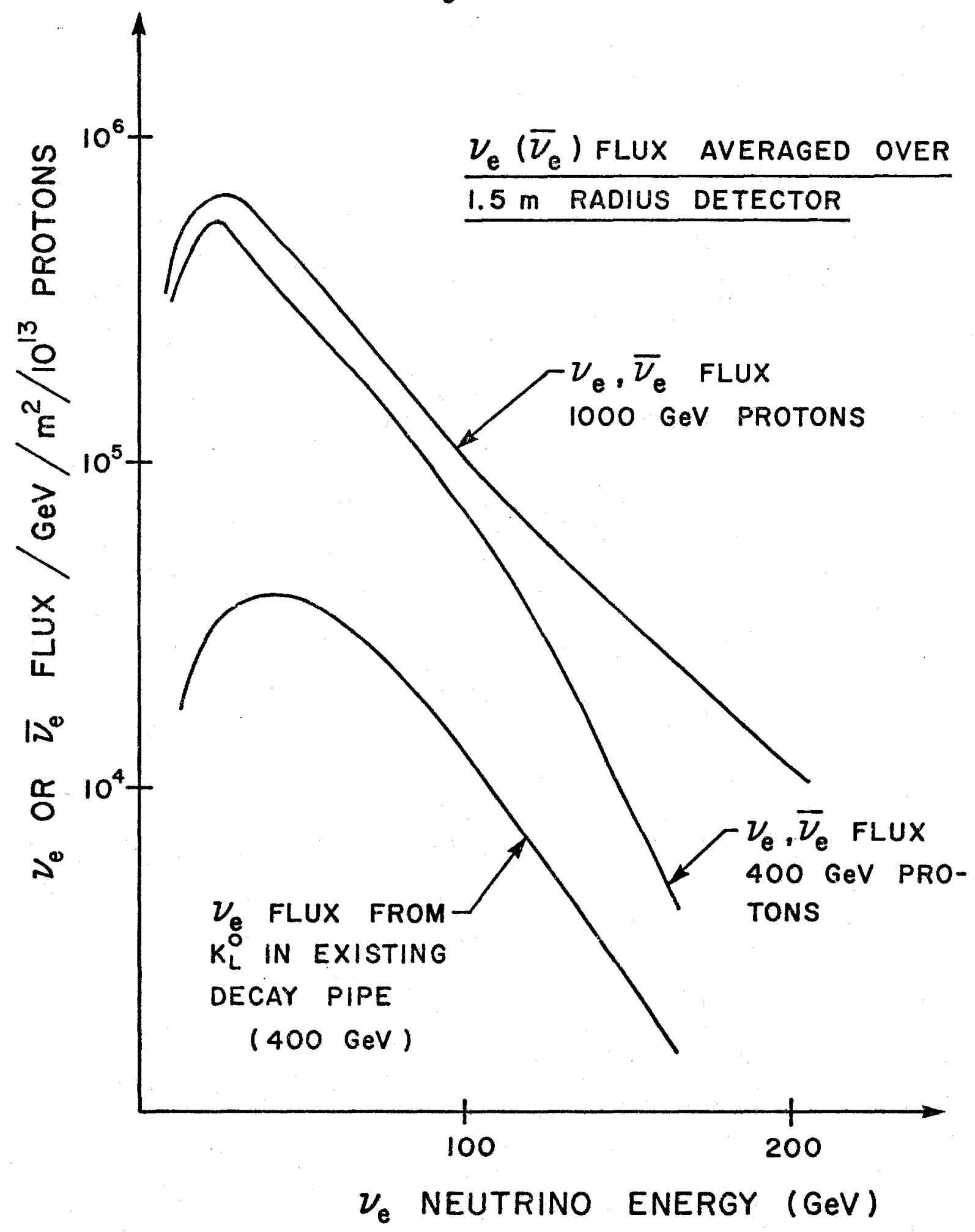




\section{$\pm 5 \mathrm{mr}$ Beam Design}
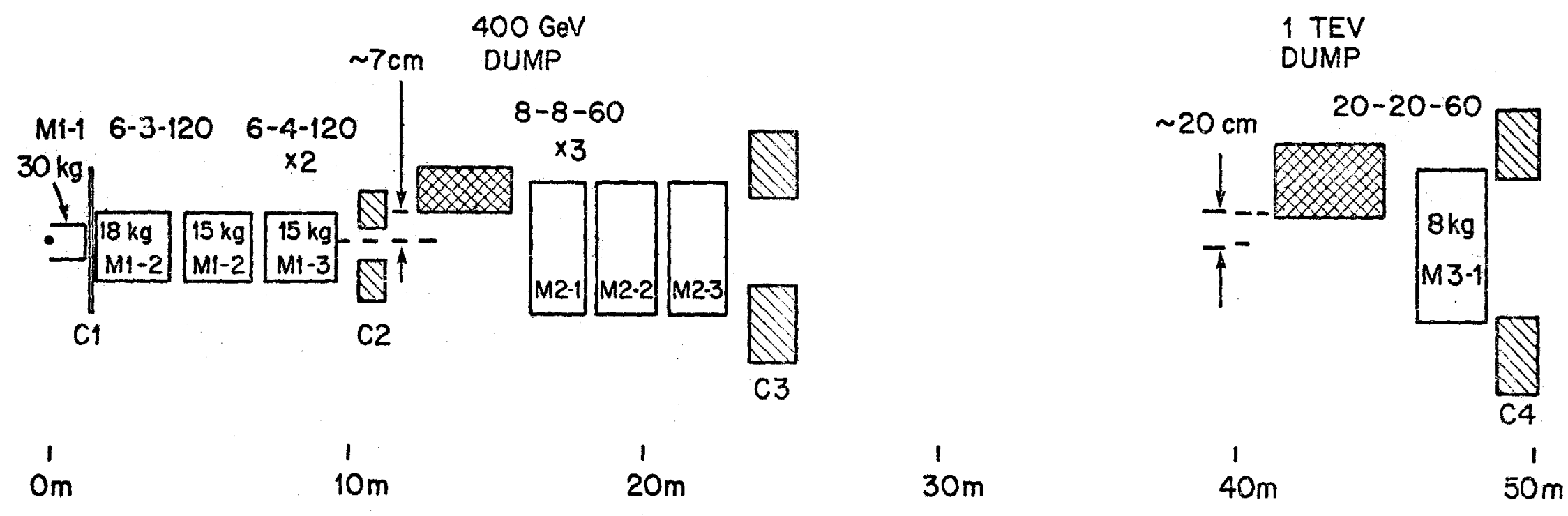

Figure 5

푸ㅇㅝㅗ

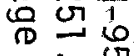

जें 


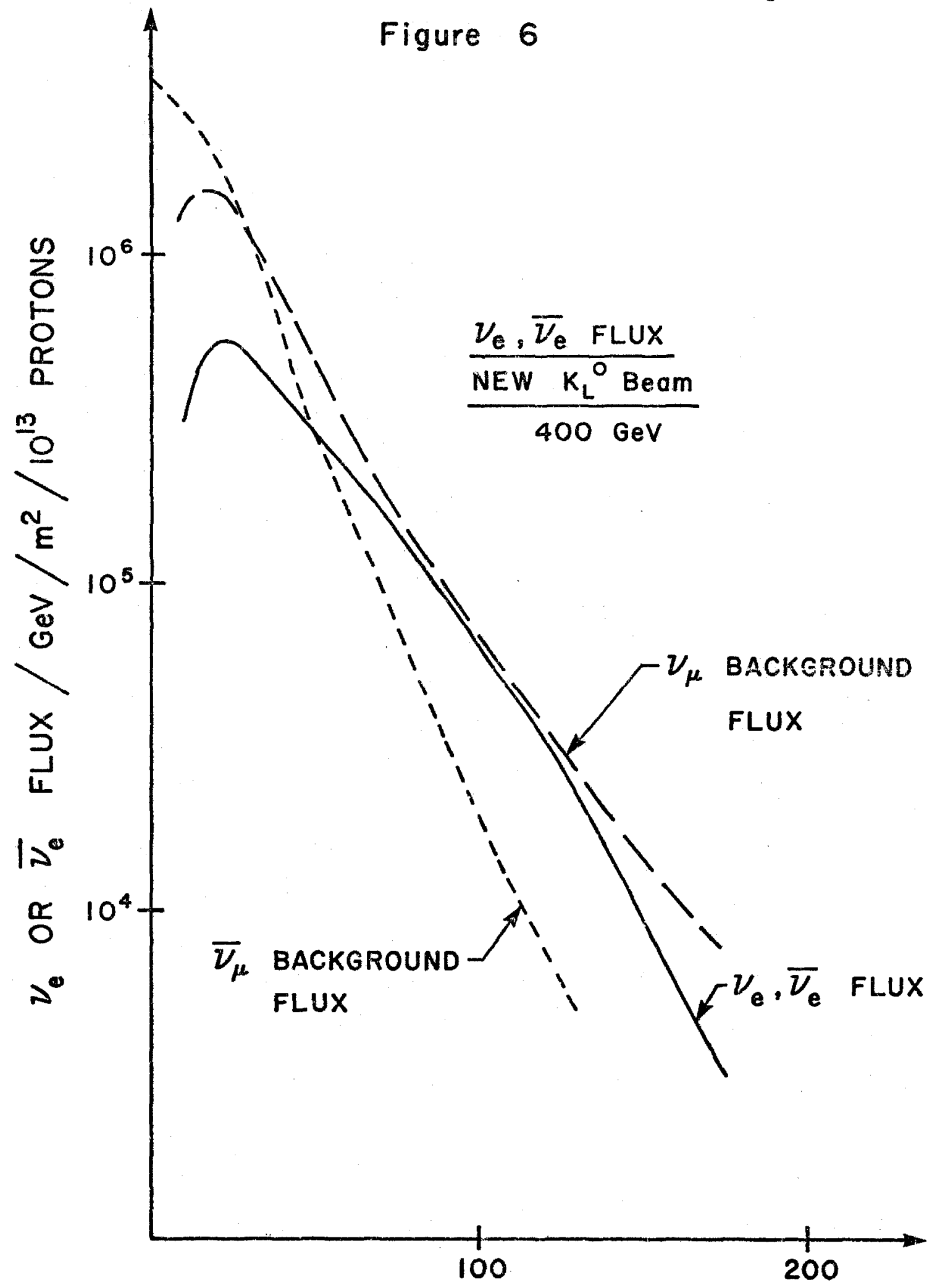

NEUTRINO ENERGY ( GeV) 


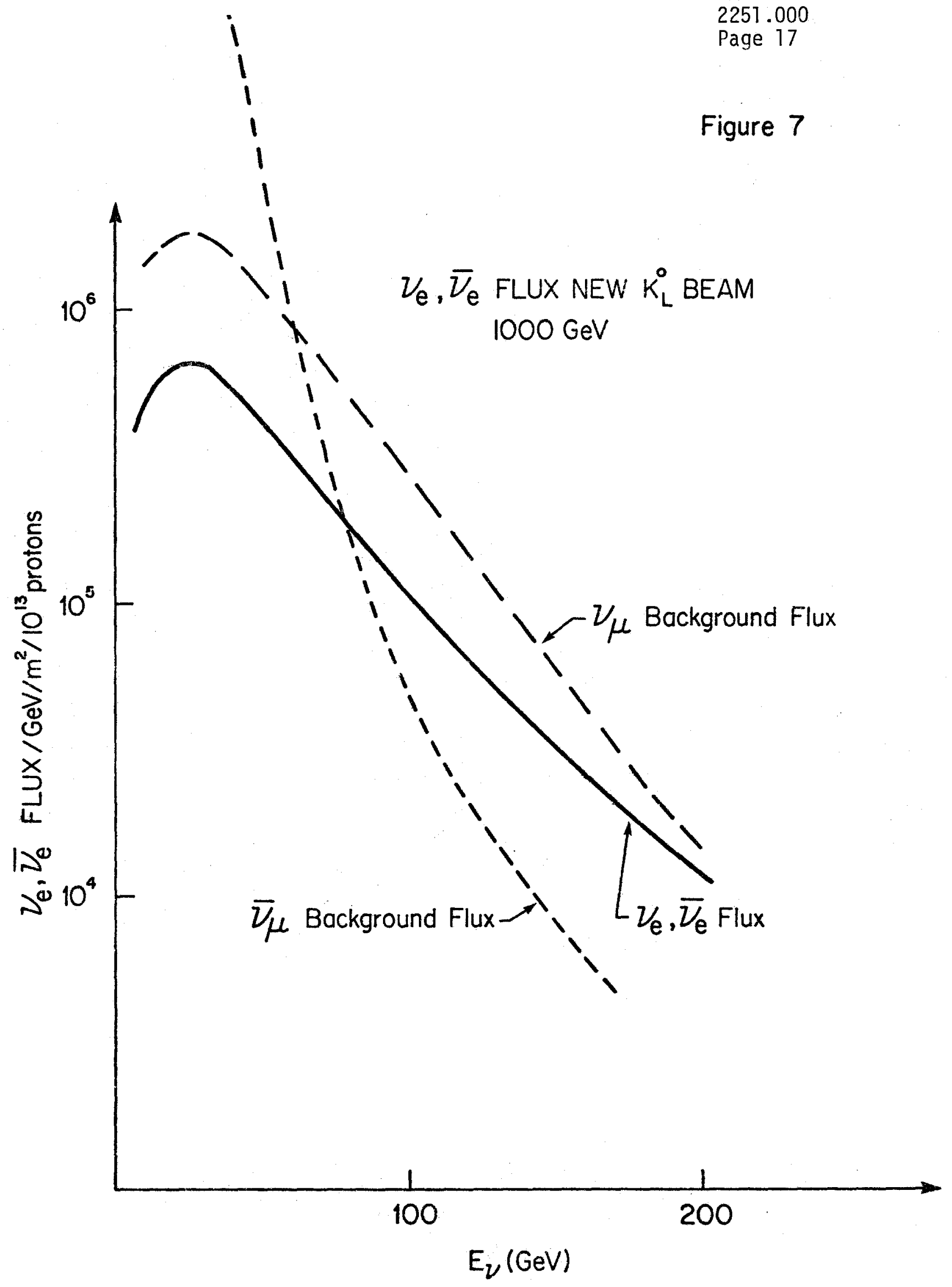


CHARM PRODUCTION CROSS SECTION Figure 8 $400 \mathrm{GeV}$ protons; $\sigma \propto A \mathrm{~A} ; \operatorname{BR}\left(D_{23}\right)=10 \%$

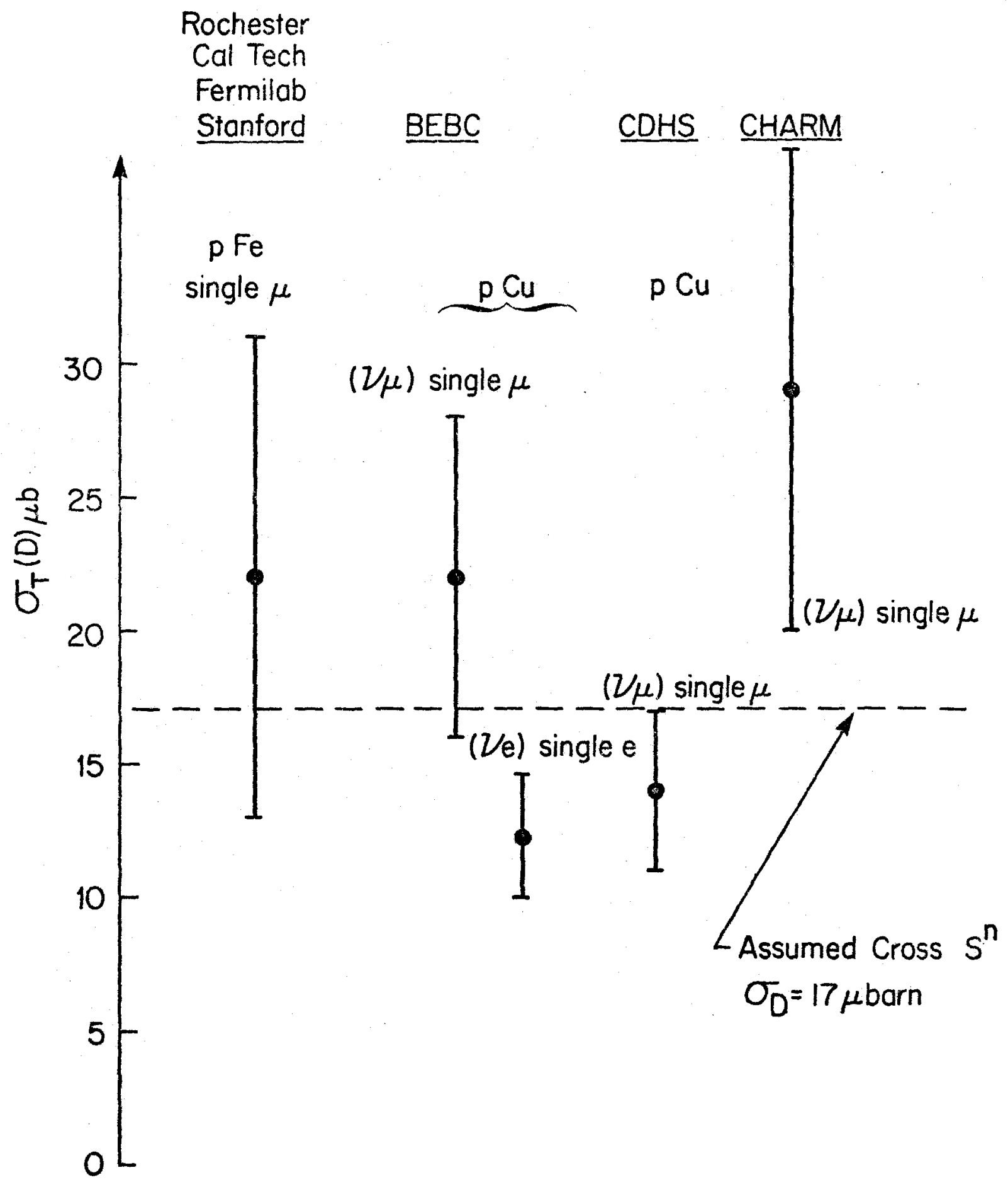




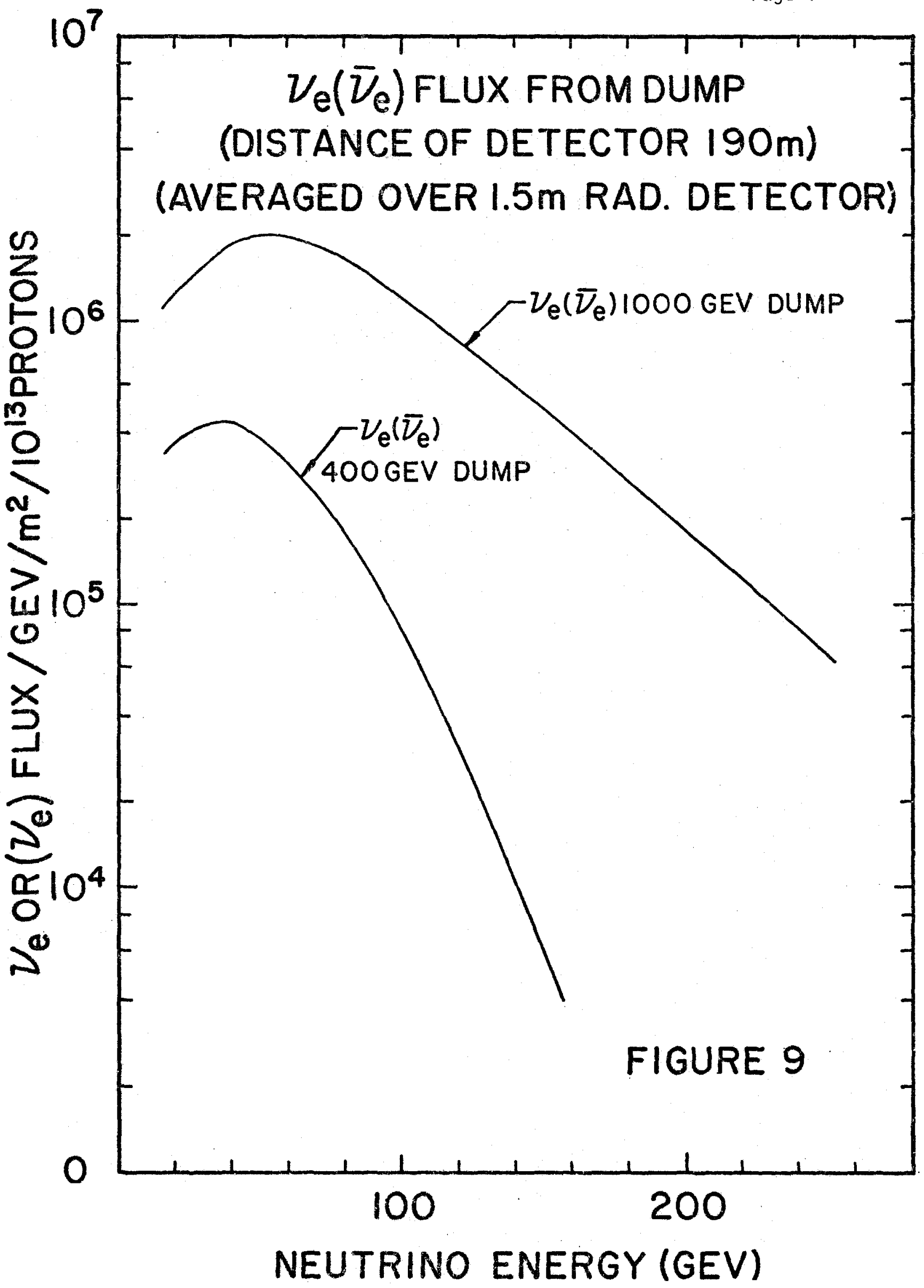




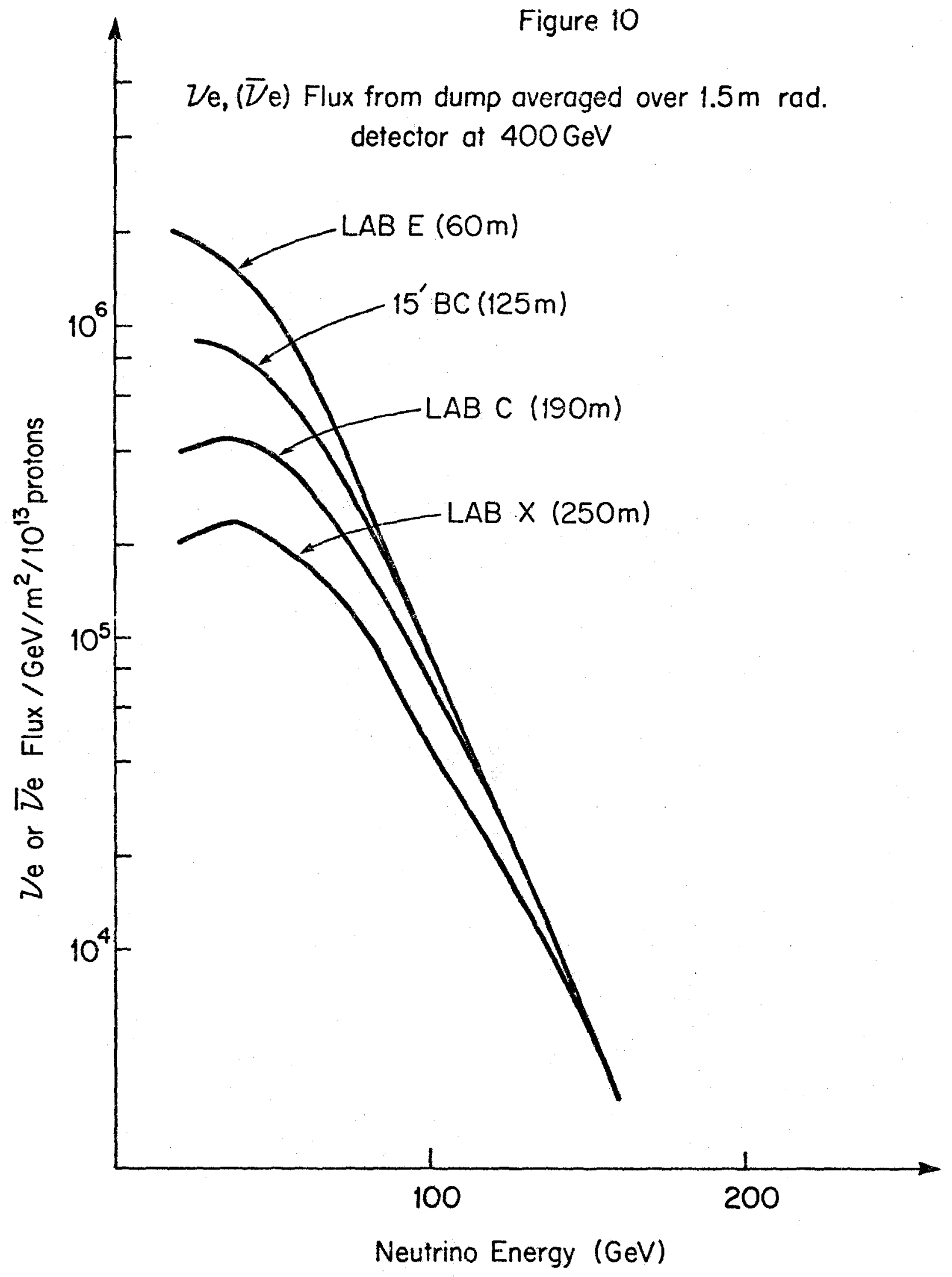




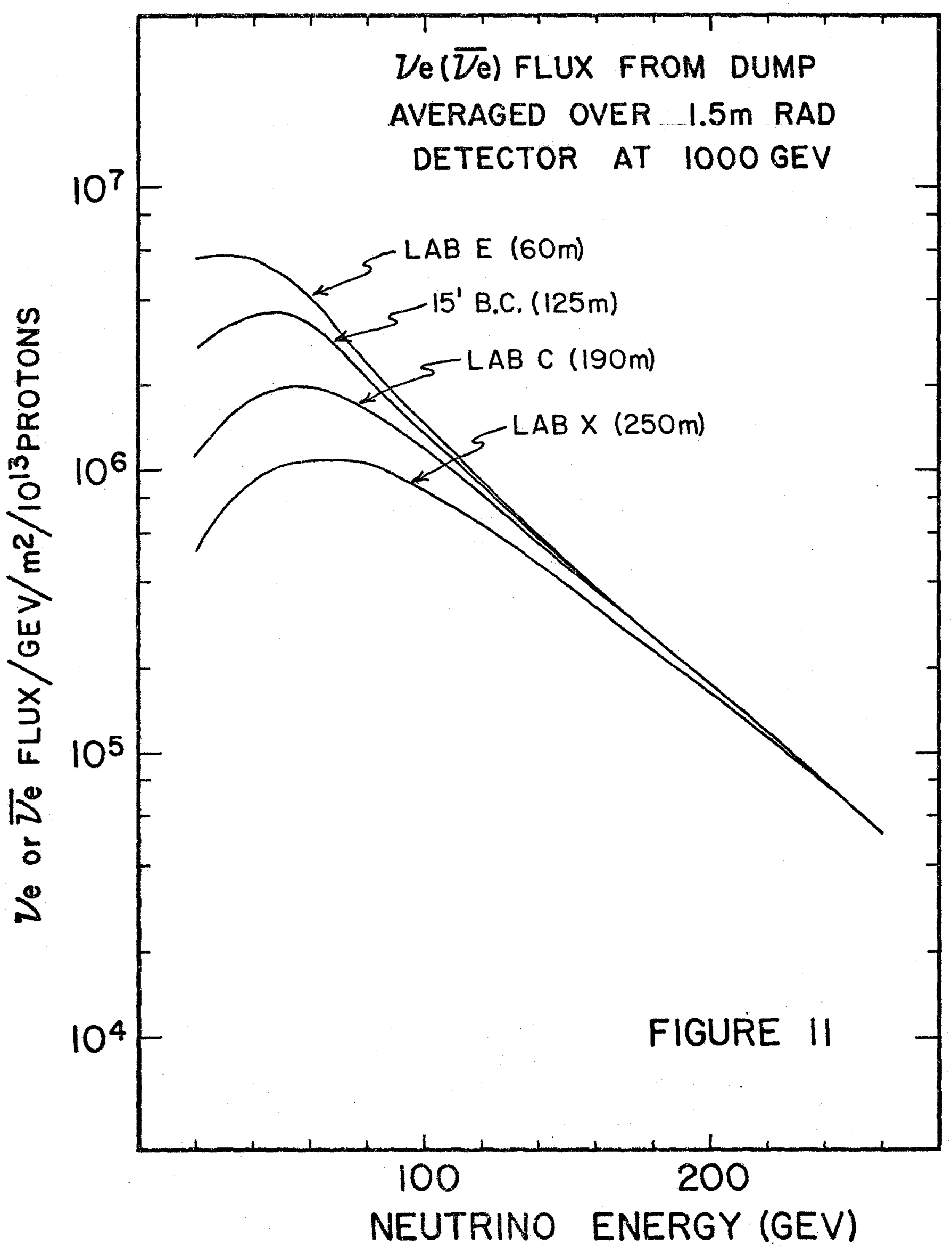




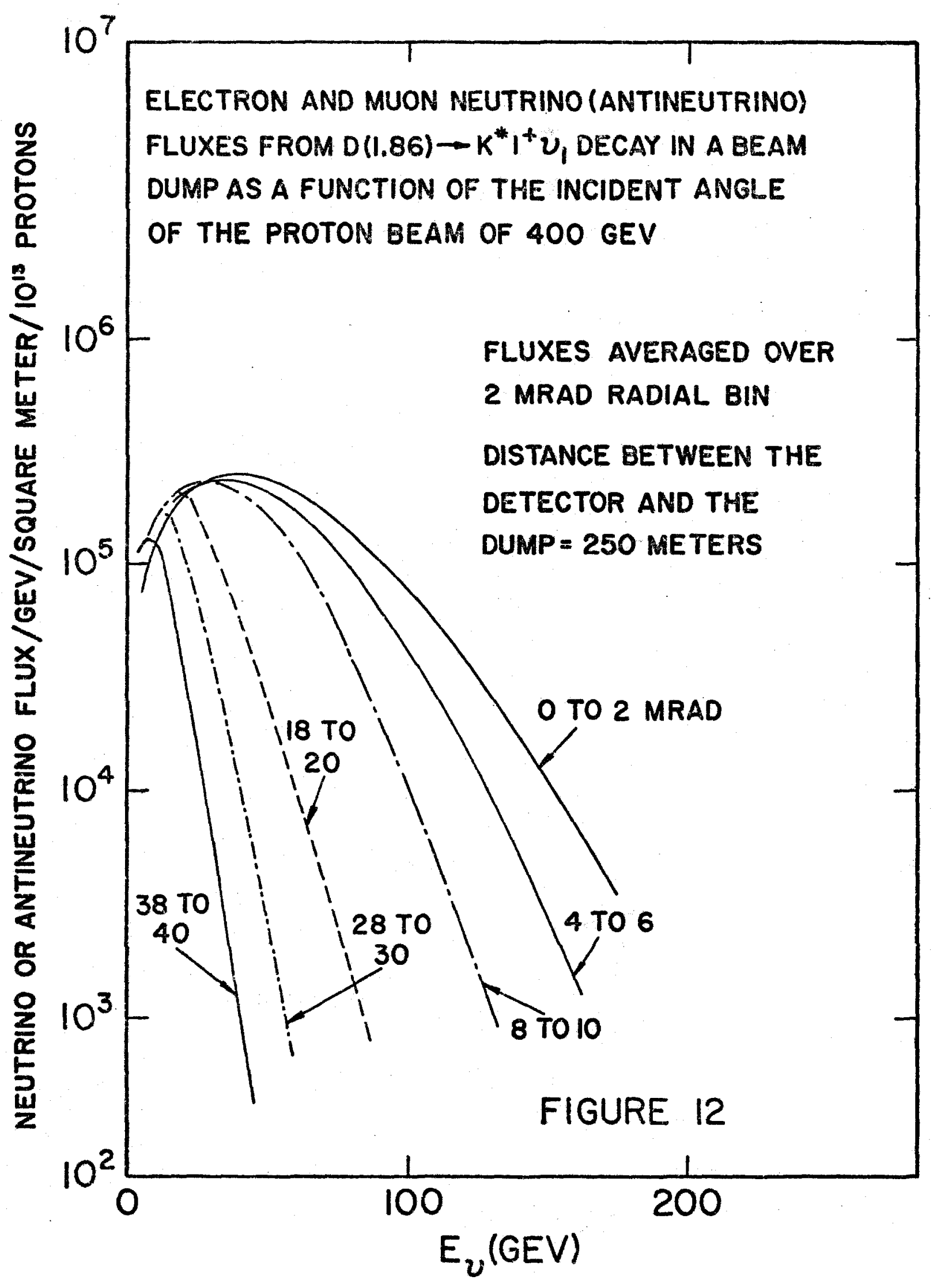


Figure 13

Electron and muon neutrino (antineutrino) fluxes from $D(1.86)+K^{*} 1^{+} \nu_{1}$ Decay in a beam dump as a function of the incident angle of the proton beam of $1000 \mathrm{GeV}$

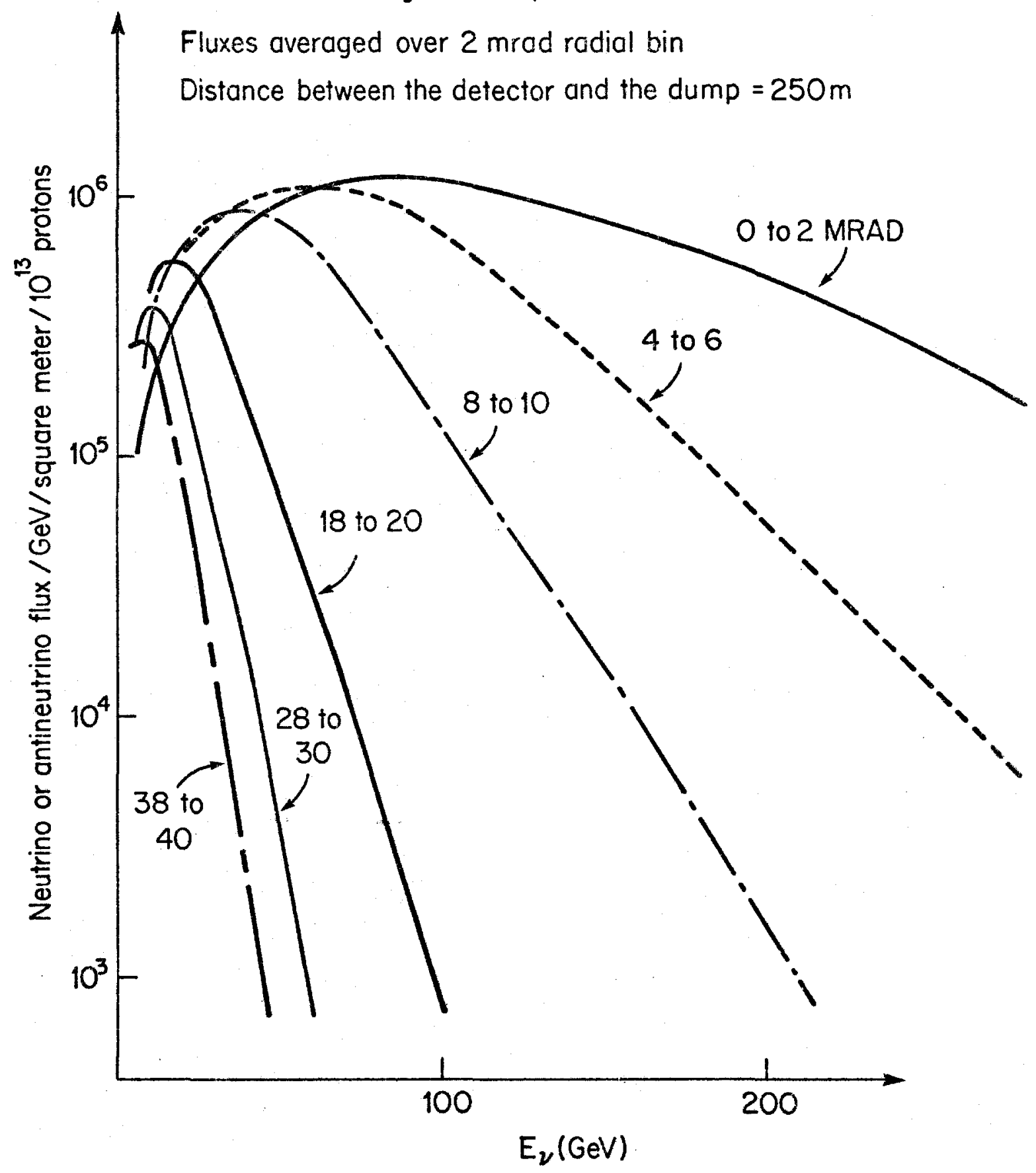




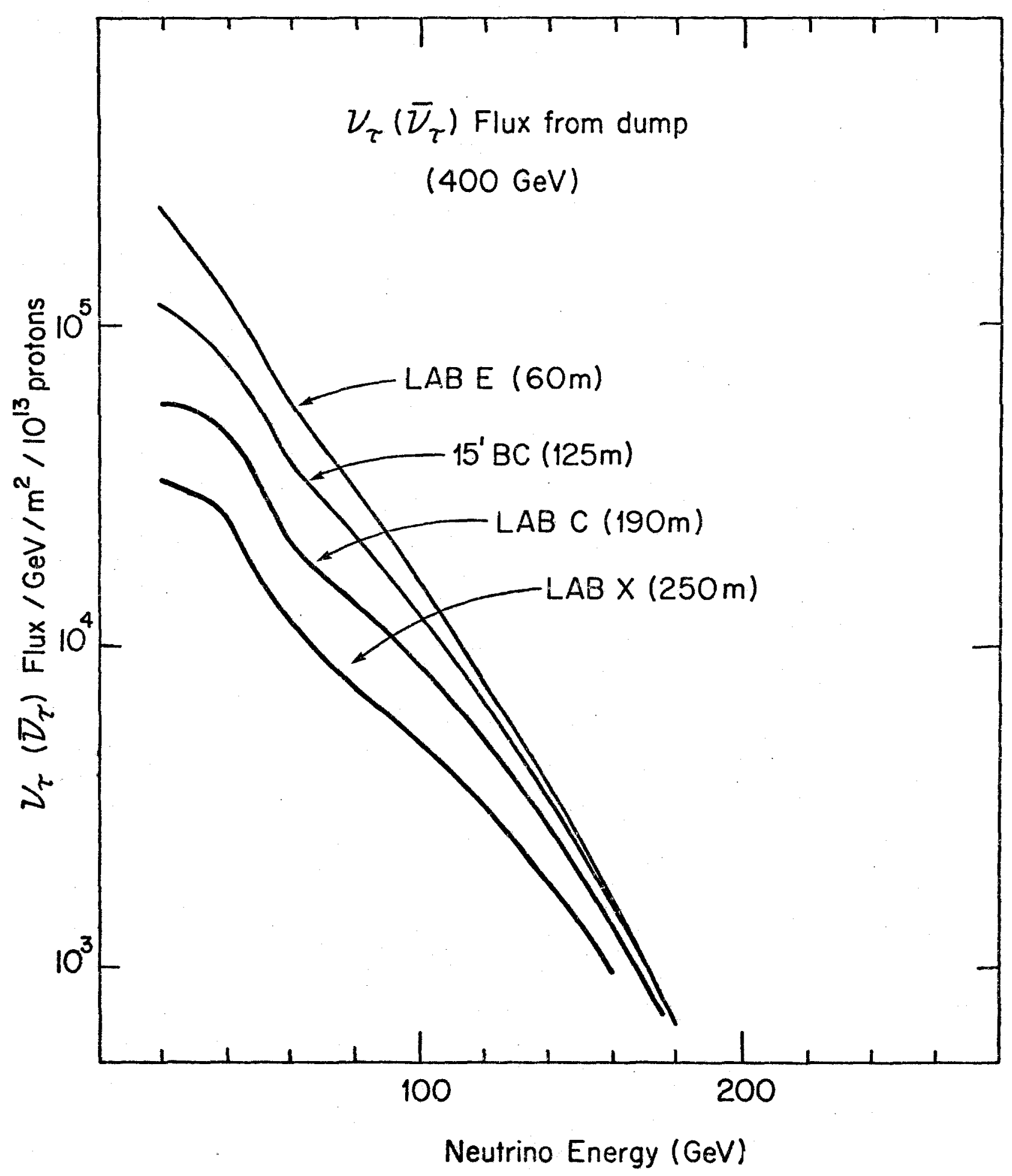

Figure 14 


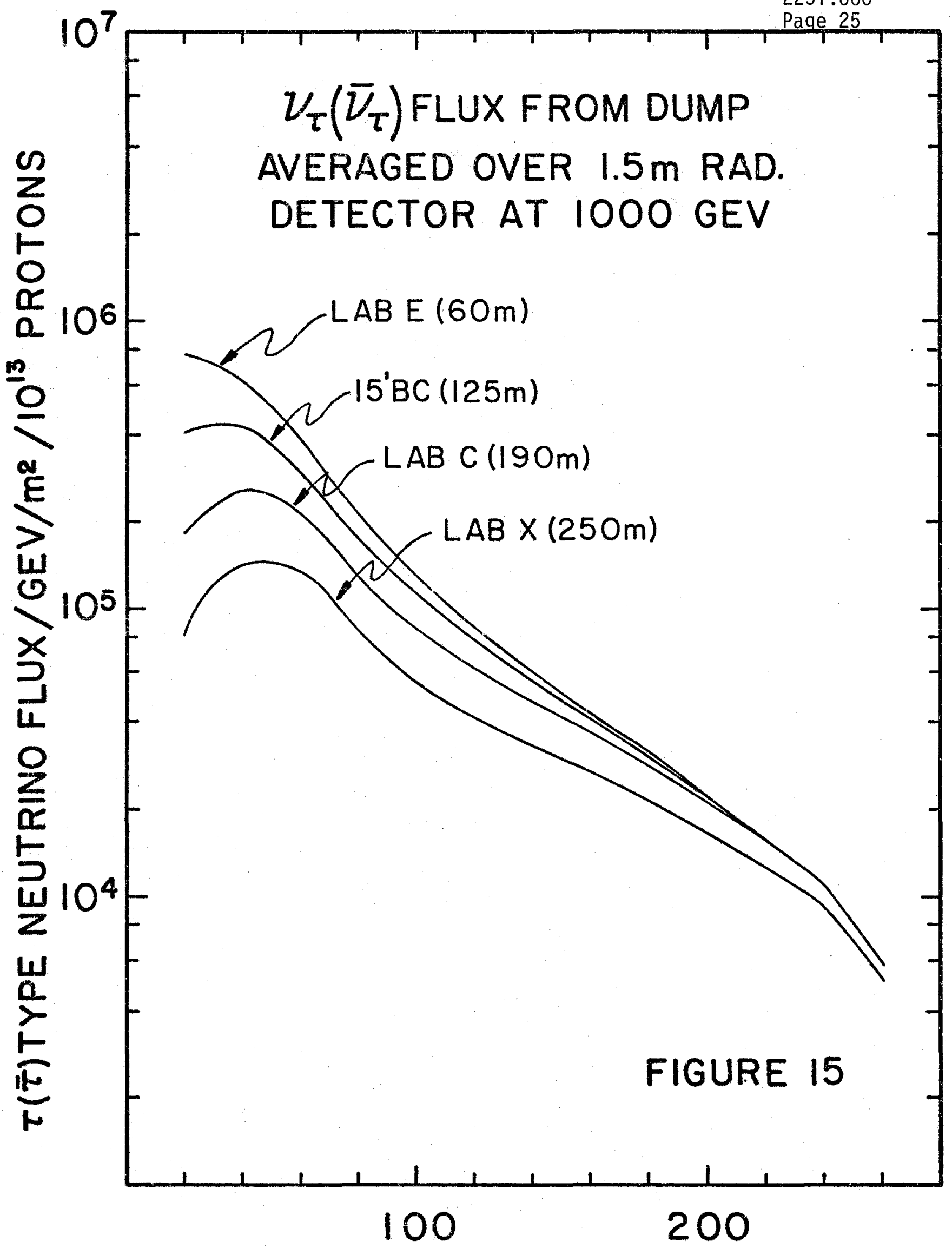

NEUTRINO ENERGY (GEV) 
Figure 16

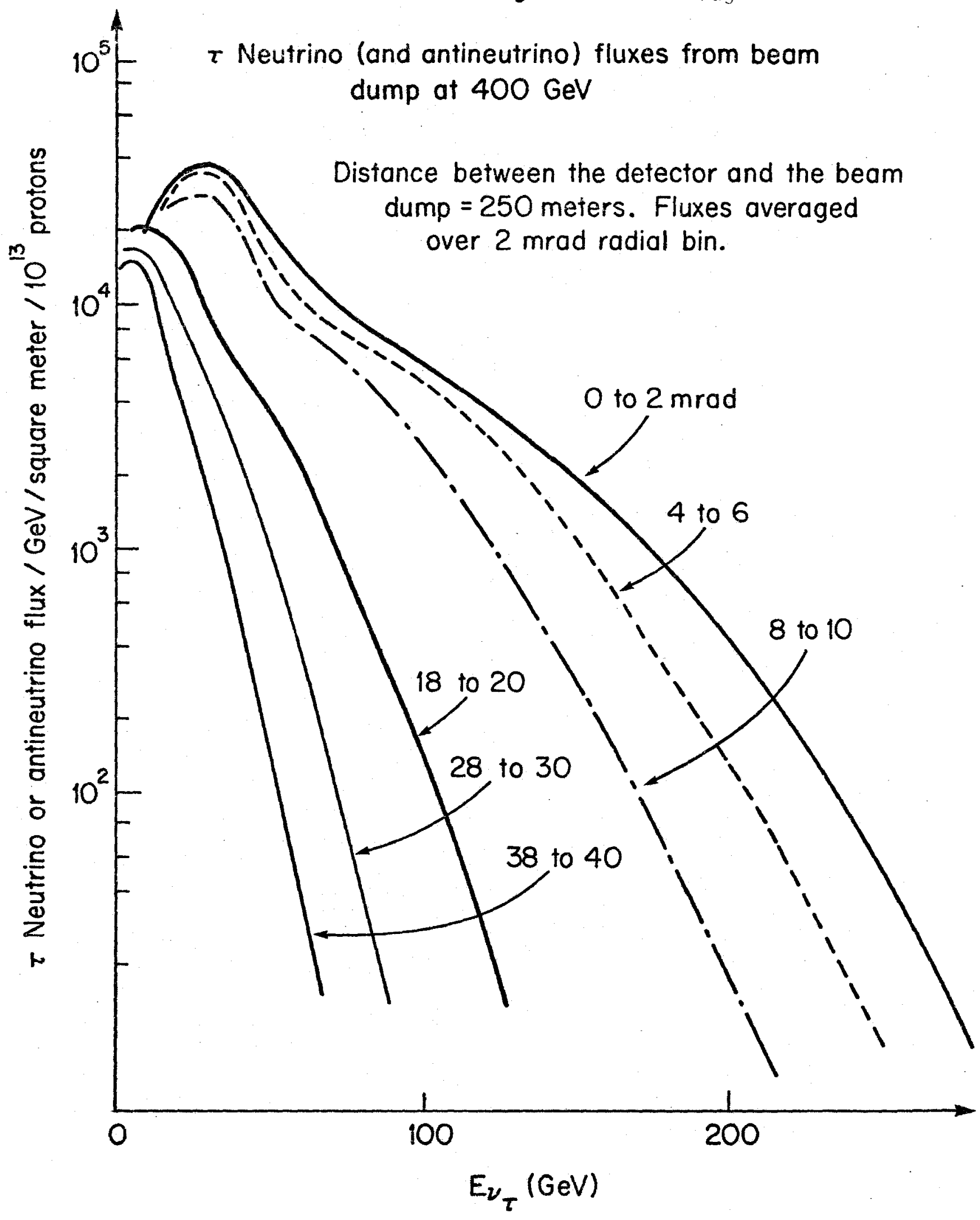


Figure 17

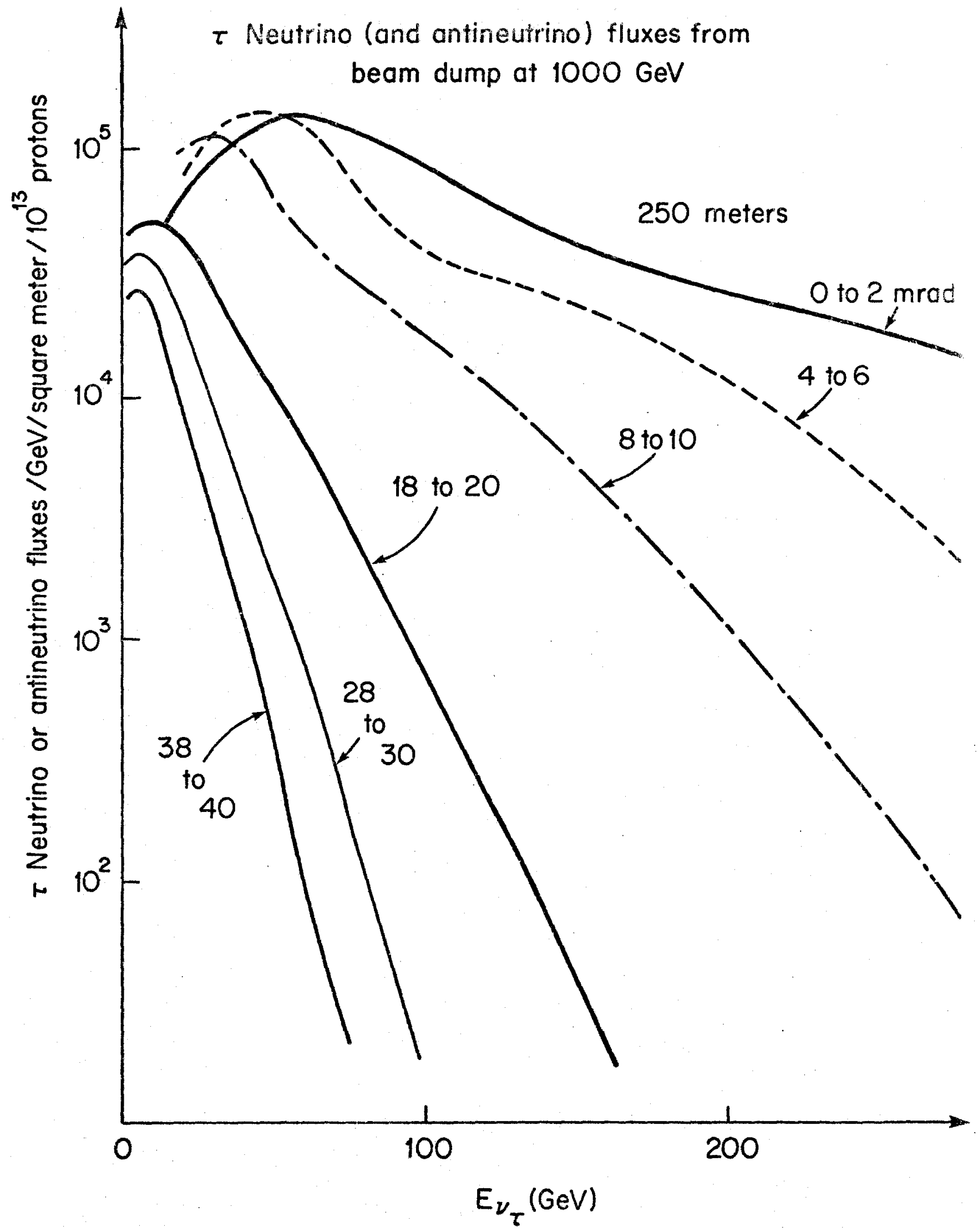




\title{
곡 Fermilab \\ TM-953-A \\ 2251.000
}

\author{
Addendum to \\ A Flavored Neutrino Beams Facility \\ S.Mor $i$ and J.K.Walker \\ June 4,1980
}

\begin{abstract}
A11 fluxes from the tungsten beam dump should be adjusted downward by a factor of 0.49 . This is due to the fact that the units should be $10^{13}$ interacting protons. The change from copper to tungsten reduces the interaction length and gives a ratio of prompt production/interaction length $\propto A / A^{2 / 3}=A^{1 / 3}$.
\end{abstract}

